\title{
INFLUENCIA DE LA COMPETITIVIDAD EN EL PROCESO DE DESARROLLO DE LA REGIÓN JUNÍN
}

\section{INFLUENCE OF COMPETITION IN THE PROCESS OF DEVELOPMENT OF THE REGION JUNÍN}

\author{
Yamill Barrionuevo Inca Roca ${ }^{10}$, Wilmer Aliaga Tabraj y Nadia Yaranga Rodríguez
}

Facultad de Administración de Empresas de la Universidad Nacional del Centro del Perú

\section{RESUMEN}

El análisis del comportamiento regional en lo económico, administrativo, social y político, ha brindado los elementos para plantear el nuevo enfoque competitivo para el desarrollo regional. De esta manera, los sectores productivos se convertirán en la base para ejecutar un conjunto de actividades coordinadas y lograr el desarrollo en los espacios geográficos reflejados en corredores económicos con la participación organizada de la población. La identificación y concreción de oportunidades de inversión va a ocupar un espacio importante en el crecimiento regional de los próximos años, particularmente en los sectores agropecuario-forestal, turismo, pesquería y artesanal, ello permitirá generar empleo necesario para enrolar a los jóvenes de la región. El crecimiento de la demanda de productos ecológicos en el mercado mundial, la tendencia al mayor consumo de alimentos de origen vegetal, la valorización de productos como: la maca, granos andinos, papa nativa, alcachofas, entre otros, otorga fortalezas a la región, ya que se producen en determinadas cuencas y sub cuencas, que presentan condiciones ecológicas y climáticas únicas. La explotación de recursos minero-metalúrgicos debe guardar relación con la conservación del medio ambiente, donde el Estado cumple el rol y la misión de supervisor, para que se cumplan determinadas reglas de juego dentro de un marco de manejo racional de recursos naturales. El estudio es el producto de un enfoque de desarrollo de los diversos sectores económicos, como un esfuerzo para proponer la misión y visión que las autoridades y pobladores en su mayoría plantearán para la región en el futuro, asociados a la identificación de oportunidades de inversión desarrollo para la producción de bienes y servicios, bajo un enfoque empresarial orientado al mercado.

Palabras clave: sectores productivos, competitividad, corredores económicos

\section{ABSTRACT}

The analysis of regional performance in economic, administrative, social and political matters has provided the elements to bring the new competitive approach to regional development. Thus, the productive sectors will become the basis for implementing a coordinated set of activities and achieve development in the geographical areas reflected in economic corridors with the organized participation of the population. The identification and realization of investment opportunities will occupy an important place in regional growth in the coming years, particularly in the agriculture, forestry, tourism, fisheries and handicrafts, it will generate employment necessary to enroll the youth in the region. The growth in demand for organic products in the world market, the trend towards increased consumption of plant foods, evaluate products like maca, grains Andean native potatoes, artichokes, among others, gives strength to the region, occurring in some basins and sub basins, which have unique ecological and climatic conditions. The exploitation of mineral and metallurgical resources must be related to

\footnotetext{
10 a_yamill@yahoo.com
} 
the conservation of the environment in which the state plays the role and mission of supervisor, to fulfill certain rules of the game within a framework of sound management of natural resources. The study is the product of a focus on development of various economic sectors in an effort to propose the mission that the authorities and people mostly arise for the region in the future related to the identification of investment opportunities development for the production of goods and services under a market-oriented business approach.

Key words: productive sectors, competitiveness, economic, corridors

\section{INTRODUCCIÓN}

Este marco nos permitirá entender los criterios necesarios sobre el cual debe basarse la competitividad de la Región en su proceso de desarrollo. Posteriormente, se desarrollarán los sectores que son competitivos y las estrategias que serían necesarias para lograr la competitividad regional y empresarial.

"Competitividad es la capacidad de un país 0 región para generar proporcionalmente más riqueza que sus competidores en el mercado mundial."

Esta definición corresponde al Instituto para la Gestión del Desarrollo- IMD - de la Universidad de Laussanne, Suiza, quien cada año emite el Reporte Mundial de Competitividad de los países, el cual es presentado en el Foro Económico Mundial.

El análisis de las estrategias competitivas más utilizadas son: costo, diferenciación 0 segmentación, su éxito relativo, la evaluación de las fortalezas y debilidades de los competidores, sus metas, objetivos, capacidades y aún más el saber quién es el competidor más importante. Identificar y aceptar el propio posicionamiento y el de los competidores, saberse líder 0 seguidor, conocer la información financiera de los competidores. Estos son los conceptos que se manejan en el mundo actual.

Para conseguir incrementos en la competitividad, se tiene que establecer el atractivo del sector, a fin de determinar si se ingresa o no a este, la altura de las barreras para el ingreso, la calidad y cantidad de los competidores existentes, el peligro de sustitución y el poder de negociación de los compradores y los proveedores. Estos, conjuntamente con el análisis de grupos estratégicos, el posicionamiento estratégico y la cadena de valor, son aportes de estudiosos como el profesor Michael Porter, cuyas obras han sido tomadas de base para la realización del presente trabajo.

En la Región Junín se debe capacitar a los cuadros gerenciales y funcionarios públicos y privados en las modernas técnicas de competitividad para que al llevarlas a sus empresas se logre la interiorización de todo el personal en el tema, a fin de alcanzar la masa crítica necesaria para formar los indispensables Centros de Competitividad.

\section{MATERIAL Y MÉTODOS}

Material

En el desarrollo del presente trabajo de investigación se han recurrido al uso de materiales, como material bibliográfico, informes, textos, así como material de escritorio y equipos como computadora, plumones, lapiceros, papeles, tableros, calculadora, cuestionarios etc.

\section{Método}

Método de Investigación no experimental o ex post-factum

La investigación no experimental es sistemática y empirica; en ella, las variables independientes no se manipulan, porque ya han sucedido; las 
inferencias de las relaciones entre las variables se realizan sin intervención o influencia directa, y se observan tal y como se han dado en su contexto natural.

En la investigación ex post-factum, el diseño se caracteriza por identificar, observar y describir las variables, sin posibilidad de manipularlas. La locución latina significa "a partir de lo ya sucedido".

Nuestra investigación es no experimental y ex post factum porque con el instrumento diseñado identificamos, observamos y describimos el plan de desarrollo turístico de la Región Junín.

\section{RESULTADOS Y DISCUSIÓN}

\section{DETERMINANTES DE LA VENTAJA COMPETITIVA REGIONAL}

Los determinantes de la ventaja competitiva regional deben reforzarse entre sí y proliferar en el tiempo para fomentar la ventaja competitiva en un sector económico determinado.

Estos atributos que, en conjunto 0 individualmente, constituyen el "diamante" de la ventaja competitiva de una región y que le permiten optar por decisiones estratégicas cada vez más sofisticadas, son:

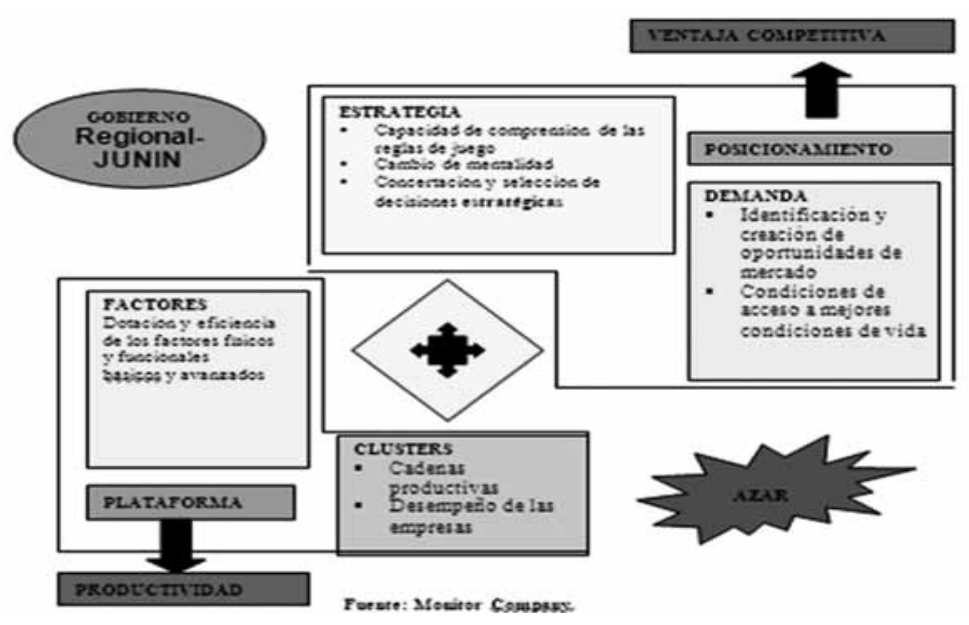

\section{CONDICIONES DE LOS FACTORES}

Constituida por los recursos humanos, la infraestructura física y técnica, los factores físicos de producción, los conocimientos, eficiencia y efectividad para su explotación y habilidades para mejorarlos.

Michael Porter los clasifica en:

Los factores básicos, que se heredan o se crean con muy poca inversión, son:

Los recursos naturales de la región: suelos, hidrología, mineros, metalúrgicos, no metálicos, etc.
Situación geográfica: ubicación geopolítica 0 estratégica.

Climas y microclimas, pisos ecológicos, etc.

Biodiversidad: flora y fauna.

Mano de obra, poca o nada especializada.

Los factores avanzados, que requieren inversiones cuantiosas y de largo plazo.

Infraestructura física, vial, portuaria, etc.

Mano de obra especializada, capacitación, conocimientos.

Soporte tecnológico para la industrialización. 
Investigación científica específica para los sectores productivos

Debido a la globalización es que los factores básicos han dejado de ser fuente de ventaja competitiva, pues las empresas pueden trasladarse hacia los lugares donde estos se encuentran. Inclusive las unidades de producción ya no se ubican sólo donde se encuentra la materia prima, sino donde el proceso productivo puede adquirir mayor nivel de agregación.

La moderna competitividad regional está basada en los factores avanzados y especializados; o sea, en la capacidad de innovación y en la creatividad de sus recursos humanos.

\section{CONDICIONES DE LA DEMANDA}

Cuando este factor está desarrollado, el consumidor regional exige que sus productos incorporen innovaciones y normas de alta calidad, útil para anticipar la demanda mundial más sofisticada.

Para ello es necesario conocer el tamaño, composición, tasa de crecimiento, niveles de exigencia y grado de sofisticación del mercado regional. Un mercado regional exigente y sofisticado en sus hábitos de consumo, estimula la innovación y se convierte en un determinante de la competitividad, pues es capaz de crear demanda mas allá de sus fronteras.

También de acuerdo a lo afirmado por Porter, "un mercado interno pequeño puede resultar estimulante para buscar mercados exteriores", como es el caso de la región Junín.

\section{EMPRESAS RELACIONADAS Y DE APOYO "CLUSTERS"}

Cuando en una Región se desarrolla un sector productivo, los proveedores, distribuidores y las empresas relacionadas con el sector, deben apoyar y reforzar mejoras e innovaciones en los productos y procesar tecnologías para un mejor manejo de canales de mercadeo. A estas empresas se les llama "clusters".

Los clúster son los que comparten o coordinan actividades comunes en su cadena de valor, como puede ser: la producción, distribución, mercadeo, servicio pos- venta, etc.

\section{ESTRATEGIA, ESTRUCTURA Y RIVALIDAD}

Se refiere a la forma en que las empresas regionales de un sector industrial son creadas, organizadas, administradas y como compiten entre ellas. Las elecciones de ventaja competitiva, posicionamiento estratégico y tecnología utilizada definen sus comportamientos en cada sector. También, las prácticas y enfoques de gerencia comunes a las empresas de un sector, determinarán su manera de competir. Estas prácticas son:

Estilo de liderazgo.

Iniciativa individual.

Sistemas de apoyo para la de toma de decisiones.

Organización administrativa y tamaño de las empresas.

Relaciones entre la alta dirección y los trabajadores.

Factores culturales, idiosincrasia y motivaciones.

Este determinante actúa como promotora de una dinámica competitiva que inyecta energía al "diamante" y produce compañías del más alto nivel mundial. Es importante la rivalidad interna, puesto que eso obliga a la mejora de los productos y a que reduzcan costos mediante la creatividad y la innovación. 


\section{ENFOQUE COMPARATIVO DE LA COMPETITIVIDAD DE LOS SECTORES ESTRATÉGICOS}

De los mapas competitivos se han extraído los siguientes puntos comunes clasificados por sectores competitivos y estratégicos, que se resume en el cuadro siguiente:

\section{Cuadro $\mathrm{N}^{\circ} 1.1$}

Junín: Comparación de Mapas de Competitividad por Sectores Estratégicos de la Región

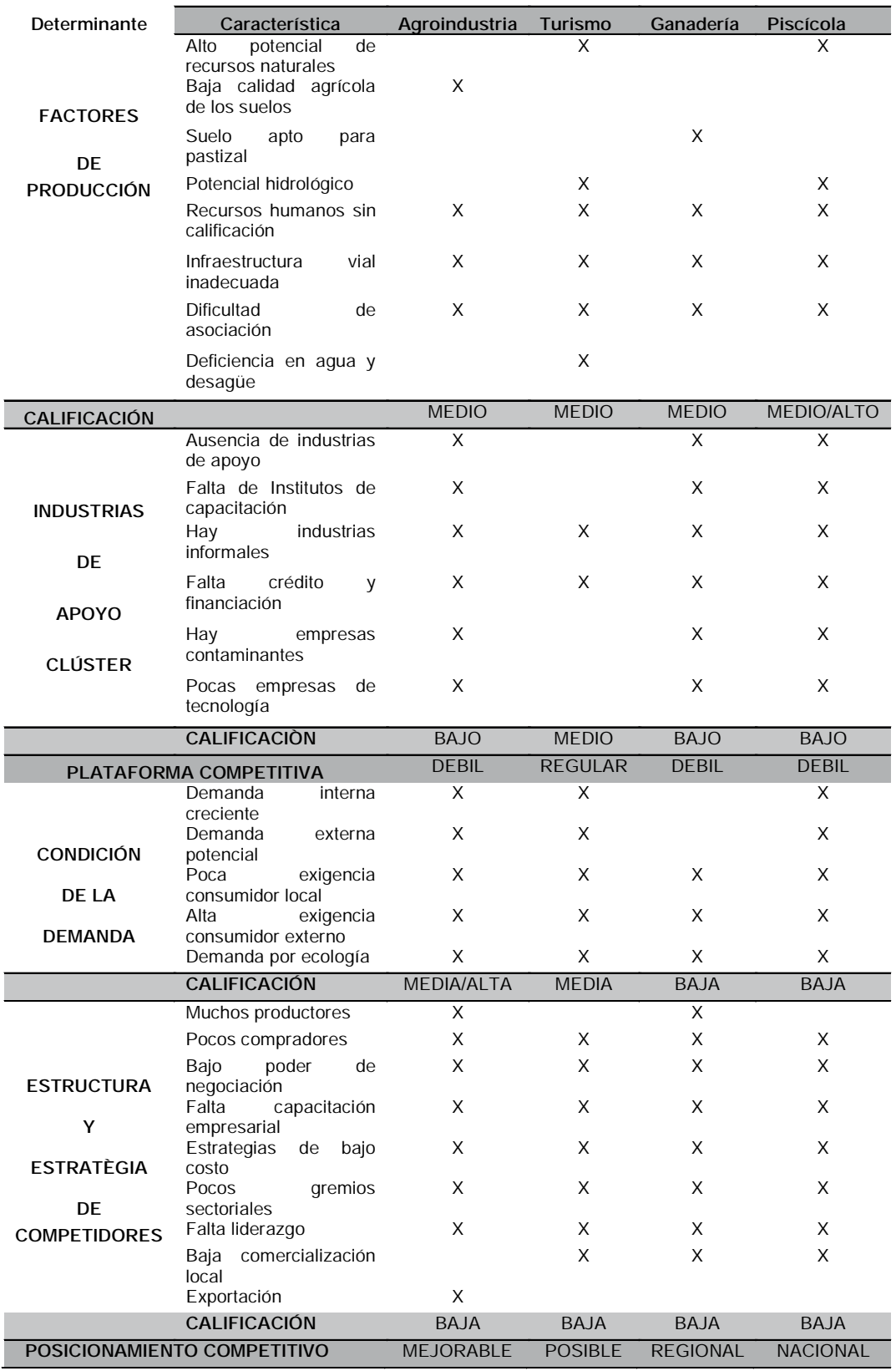




\section{Prospectiva Universitaria}

Fuente: Elaboración del Equipo de investigación.

Lo que se puede apreciar del cuadro anterior es lo siguiente:

El sector Agroindustria tiene una base competitiva débil, fundamentalmente por la baja calidad de sus suelos y las dificultades asociativas de su recurso humano, sumadas a la ausencia de industrias de apoyo, pero puede lograr su posicionamiento si se adecuan estrategias que mejoren su producción, comercialización y estrategias de mercado, para lo cual se requiere un liderazgo claro. Por lo tanto, puede generar ventaja competitiva, siempre y cuando se maneje el tema ecológico, el cual es un factor de diferenciación importante.

El sector Turismo también carece de una sólida base competitiva, por el problema de infraestructura física y vial y sus recursos humanos poco capacitados, pero su posicionamiento también permite posibilidades ciertas de desarrollo, basado en sus recursos naturales, en el campo del Ecoturismo y el Turismo de Naturaleza Aventura principalmente. Esta será su ventaja competitiva.

La ganadería lechera tiene una base competitiva débil debido a la poca capacidad de asociación de los productores, ausencia de industrias de apoyo y baja capacitación, que le resta poder de negociación y mejores perspectivas de desarrollo empresarial. La atomización de productores y sus nulas estrategias comerciales, han determinado que no se considere a la región como productora de leche. Sin embargo, habiendo sido el Mantaro una cuenca lechera reconocida, es posible recuperar ese estado en los siguientes años, para lo cual se debe implementar estrategias específicas sobre la base del repoblamiento y utilización de ingeniería genética, utilizando al máximo los ingentes pastizales de la región.

El sector Piscícola tiene muchos recursos hidrológicos aún sin explotar, pero la ausencia de industrias de apoyo, poca o nula capacitación piscícola y baja tecnología determinan una plataforma competitiva débil, que no permite que este sector importante alcance el nivel que debe tener en la economía regional. La demanda de trucha en el ámbito regional, nacional y mundial, es la base de la consideración estratégica de este sector, el cual debe su bajo posicionamiento en el mercado, a que no existe estrategia de producción y comercialización y todo es a nivel artesanal, salvo el caso de algunas piscigranjas como Ingenio y Los Andes. En la medida que se den estrategias adecuadas, capacitación y tecnología, aprovechando las exigencias del mercado interno y externo sobre este alimento, el sector piscícola se convertirá en importante engranaje de la economía de Junín.

La competitividad debe ser liderada en forma compartida por todos los sectores involucrados.

La competitividad es un proceso natural y con dinámica propia, que requiere del desarrollo de su mercado interno para tener una plataforma que le permita impulsar sus productos al extranjero.

Existe una gran demanda en el mercado internacional, sobre todo por productos ecológicos y frescos, como base alimenticia y nutricional de las nuevas generaciones, ante la arremetida de enfermedades como el cáncer y otras pestes.

Junín tiene ventajas comparativas basadas en su ubicación estratégica, sus recursos naturales o biodiversidad y sus métodos tradicionales de cultivo.

Las ventajas comparativas no son suficientes, ya que lo que realmente importan son las condiciones de factor 0 recursos avanzados, tal como la mano de obra especializada y calificada, la infraestructura vial, modelos asociativos, etc.

Las ventajas comparativas se convierten en ventajas competitivas mediante estrategias en 
las que deben participar todos los sectores, el público en su rol facilitador y el privado como financiador.

\section{REFERENCIAS BIBLIOGRÁFICAS}

10 Documentos con información estadística del departamento de Junín, según varias fuentes: INEI, Ministerio de Agricultura-Región Junín, etc.

Aco Pascual, Hacia una Cultura Creativa y Democrática. Inédito, Lima 2003.

Banco Central de Reserva, Su historia y la economía del Perú; 1821-1992. BCR, Lima 2000

Banco de Datos Estadísticos, Cámara de Comercio de Huancayo. 2008.

Barcelli, Agustin, Breve historia económico social del Perú. Edit. Jatunruna, Lima 2002.

Caracterización de los Recursos Naturales Renovables para el Alivio a la Pobreza en la Sierra; INRENA.2007

Chiroque, Sigfredo, Mapa de la Pobreza Educativa en el Perú. Instituto de Pedagogía Popular, Lima. 2000.
Contreras Carlos - Cueto Marcos, Historia del Perú Contemporáneo. PUCP-IEP, Lima.2009.

Cotler, Julio, Clases, Estado y Nación en el Perú. IEP, Lima. 2008.

Cuencas hidrográficas. Documento no publicado en revisión

Diagnóstico de la Región Central; INP. 2007.

Diagnóstico de los valles del Mantaro y Tarma; PN-PMI. 2008.

Diagnóstico Socio Económico de la provincia de Satipo; 2004

Diagnóstico Socio Económico para el Reforzamiento de los Centros Poblados en el valle del Mantaro; INP. 2007.

Documentos con información estadística del departamento de Junín, según varias fuentes: INEI, Ministerio de Agricultura-región Junín, etc.

Economía Ecológica. Por Fac. de Ciencias Humanas y Económicas, Postgrado en Economía. Colombia. 2009. 